\title{
El turismo activo en la comunidad. Relación entre impactos percibidos por los residentes y apoyo al desarrollo del sector
}

\section{Active tourism in the community. Relationship between perceived impacts on residents and support for sector development}

\author{
Rómulo JacoboGonzález-García*, María Huertas González-Serrano y Daniel Ayora-Pérez \\ Departamento de Educación Física y Deportiva, Universidad de Valencia (España)
}

\begin{abstract}
Resumen: La relación entre el sector turístico y el deporte ha crecido en importancia, tanto a nivel académico como profesional. Asimismo, los residentes tienen un importante papel a la hora de armonizar el desarrollo de la actividad en la comunidad, sobre todo en aquellas poblaciones donde la actividad turística es el sector predominante. El objetivo de este estudio es analizar qué impactos asociados al turismo activo son percibidos por los residentes y cuáles son los mejores predictores del apoyo al desarrollo de este tipo de actividad en la comunidad. Además, se ha realizado un análisis descriptivo de los ítems correspondientes a las percepciones de los residentes sobre los impactos asociados al turismo activo, así como su apoyo al desarrollo del mismo en la localidad. Resulta consecuente, la importancia en la evaluación de estos impactos por parte de los organismos encargados de la planificación, organización y gestión del sector.

Palabras clave: Percepción de los residentes; turismo activo; impacto social; apoyo de los residentes.
\end{abstract}

Abstract: The relationship between the tourism sector and sport has grown in importance, both academically and professionally. Residents also have an important role to play in harmonizing the development of the activity in the community, especially in those towns where tourism is the predominant sector. The objective of this study is to analyse which impacts associated with active tourism are perceived by residents and which are the best predictors of support for the development of this type of activity in the community. In addition, a descriptive analysis of the items corresponding to residents' perceptions of the impacts associated with active tourism has been carried out, as well as their support for its development in the area. Consequently, the importance of the assessment of these impacts by the bodies responsible for planning, organising and managing the sector is significant.

Key words: Residents' perceptions; active tourism; social impact; residents' support.

\section{Introducción}

El concepto de relación entre deporte y turismo ha aumentado en importancia en los últimos años, convirtiéndose en un campo académico de estudio, además de un producto turístico cada vez más popular. (Alguacil, Pérez-Campos, Aguado-Berenguer y Boquera, 2016). Es por ello la consecuente importancia en la evaluación de los impactos asociados a la actividad turística por parte de todos los organismos encargados de la planificación, organización y gestión del sector, siendo la percepción de los residentes sobre los principales impactos asociados al sector uno de los agentes fundamentales en los que se debe indagar (Karadakis, 2012).

Esta implicación de los residentes en el desarrollo local del sector turístico, conforma una fuerte influencia con el nivel de apoyo y actitud hacia turismo y los turistas (Hall y Page, 2014). Por ese motivo, cuándo los residentes están implicados en actividades de la comunidad tienden a ser más benévolos hacia el desarrollo y los cambios en la localidad (Woo, Uysal, y Sirgy, 2018). Sin embargo, a pesar del creciente interés académico en los últimos años por el análisis de las percepciones

Dirección para correspondencia [Correspondence address]: Rómulo Jacobo González-García. Departamento de Educación Física y Deportiva Universidad de Valencia (España). E-mail: romulojgg@hotmail.com de los residentes de la comunidad (González-García, Parra, González-Serrano, y Añó-Sanz, 2016), aún son escasas las evidencias científicas sobre las variables que predicen el apoyo al deporte como fomento del turismo (Rasoolimanesh, Ringle, Jaafar y Ramayah, 2017). En consecuencia, el objetivo de este estudio es analizar qué aspectos relacionados con los impactos asociados al turismo activo son los mejores predictores del apoyo al desarrollo de este tipo de actividad en la comunidad.

\section{Tipos de impactos}

Los impactos asociados con el turismo normalmente han sido investigados desde una perspectiva económica y ambiental, conjuntamente, los impactos socioculturales asociados a esta actividad también son frecuentemente observados (Chandralal, 2010). Por otro lado, las investigaciones a menudo coinciden en calificar los impactos como económicos, ambientales y sociales (Almeida-García, Balbuena-Vázquez y Cortes-Macías, 2015). Por su parte, Hritz y Ross (2010) en su trabajo sobre los impactos percibidos por los residentes de Indianápolis (EE.UU.) señalaron que los impactos asociados al turismo deportivo, pueden constituir cuatro factores: beneficios eco- 
nómicos, sociales y ambientales, así como impactos negativos globales.

Sin embargo, no todas las comunidades perciben los impactos del turismo de la misma manera. Ward y Berno (2011) encontraron que los residentes, generalmente, no suelen estar informados sobre los diferentes tipos de turistas que visitan su localidad y, por lo tanto, este hecho dificulta la opinión sobre los tipos de impactos que puede tener la población. La actividad turística, incluyendo específicamente la actividad física y el deporte, tiene un potencial significativo para enriquecer las vidas de los residentes de la comunidad, proporcionando oportunidades sociales y recreativas adicionales, entre otras circunstancias (Gibson 2017).

Como señalan Bartolomé, Ramos y Rey-Maquieira (2009), el impacto del turismo en las comunidades depende del tipo de turismo practicado (individual, grupal, itinerante, etc.), del propósito del viaje (cultural, de sol y playa, rural o de aventura), así como del nivel de desarrollo económico de la localidad que acoge la actividad turística.

En términos económicos, el turismo adopta una forma de consumo de bienes y servicios y, por lo tanto, tiene un impacto directo e indirecto en el empleo y las oportunidades empresariales dentro del sector. Los impactos asociados al turismo activo han tenido un impacto significativo en la relación a estos impactos (Sánchez-Fernández; Salgado-Barandela, Rodriguez-Rodriguez y Barajas-Alonso, 2016), incidiendo en su presencia sobre las comunidades que desarrollan este tipo de actividad.

El impacto cultural que tiene el turismo sobre una comunidad trasciende en las experiencias que determinados productos pueden generar sobre actividad turística. Por su parte, el deporte representa un paradigma notable en la atracción turística cultural (Higham y Hinch, 2009). Los impactos culturales asociados al turismo activo han sido examinados desde los estímulos que ejercen en el desarrollo de nuevas actividades o la conservación de bienes culturales (Jaafar, Noor y Rasoolimanesh, 2015), que puede emanar hacia un incremento en el orgullo y la identidad de los residentes (McGehee y Andereck, 2004).

En cuanto al impacto político-administrativo de la actividad turística en una comunidad, existen impactos positivos como una mayor representación de los intereses colectivos, así como una mayor contribución al desarrollo sostenible (Dumitru, 2012) o negativos, como una política ineficaz, generando una excesiva burocracia y autopromoción política (Saha y Yap, 2014).

Por su parte, el impacto social en la comunidad asociado al turismo activo está asociado a cambios en las normas y valores, tanto morales como sociales (Weed y Bull, 2012). Asimismo, Balduck, Maes y Buelens (2011) definen los impactos sociales como aquellos en los que el turismo y los efectos asociados con los viajes cambian los patrones de comportamien- to y los valores individuales, así como los estilos y la calidad de vida de una localidad.

El sector turístico, en especial en el sector del turismo activo, cuyas actividades se desarrollan en entornos naturales, tiene una gran responsabilidad en su relación con el medioambiente. A veces, estas actividades pueden ser muy agresivas con los recursos naturales si no se realizan en los lugares adecuados y con las medidas necesarias para asegurar que no causen un impacto negativo en el entorno (Dyer, Gursoy, Sharma, y Carter, 2007).

\section{Apoyo}

Comprender el apoyo de los residentes locales al desarrollo turístico es esencial para los gobiernos locales, los responsables políticos y las empresas, porque el éxito y la sostenibilidad de cualquier actividad depende del apoyo activo de las poblaciones locales (Nunkoo y So, 2016). Por esta razón, los investigadores han explorado los factores que podrían influir en estos impactos percibidos y el consiguiente apoyo al desarrollo. Asimismo, Gursoy, Chi y Dyer (2010) señalaron que el apoyo y la participación de los residentes locales es crucial para la sostenibilidad de la industria turística, por lo tanto, los residentes perciben los efectos positivos del turismo más intensamente que los negativos, lo que ayuda a explicar su apoyo al desarrollo turístico. De esta manera, conseguir el apoyo de los residentes podría convertirse en una tarea clave para los gestores responsables de la planificación y el desarrollo del turismo.

\section{Método}

\section{Participantes}

En esta investigación participaron un total de 647 residentes en Gran Canaria con edades comprendidas entre los 18 y los 86 años. La edad media ha sido de 38.13 años (DT=15.92), siendo la distribución por sexo de un $46.4 \%$ de hombres y un 53.6\% mujeres. La isla de Gran Canaria (España) tiene una población de 856.990 habitantes (2017). El error de muestreo es del $5 \%$ con un nivel de confianza del $95 \%$ para la situación más desfavorable de $\mathrm{p}=\mathrm{q}$ para toda la muestra en su conjunto.

\section{Instrumento}

El cuestionario estaba compuesto por un total de 21 ítems adaptados de otros estudios previamente validados (Delamere, 2001, González-García, Añó-Sanz, Parra-Camacho y Calabuig-Moreno, 2018) y evaluados por una escala de tipo Likert de 5 puntos. La fiabilidad se determinó mediante el alfa de Cronbach obteniendo un coeficiente de 0,79 para la escala de impactos económicos, 0,74 en los impactos cultu- 
rales, 0,81 en los impactos político-administrativo, 0,78 en los impactos social y 0,85 en los impactos medioambientales, así como una puntuación de 0,84 para la escala que mide el apoyo hacia el desarrollo de la actividad turístico activo en la comunidad.

\section{Procedimiento}

Los datos fueron recogidos entre los meses de febrero y mayo de 2017 a través de una encuesta estructurada y autoadministrada, mediante un muestreo intencional o de conveniencia. Los análisis estadísticos se llevaron a cabo mediante el paquete estadístico SPSS (Statistical Package for the Social Science, Versión 24). Asimismo, este mismo programa informático ha sido utilizado para realizar el análisis de regresión lineal múltiple. Por otro lado, para analizar la unidimensionalidad de la escala de apoyo, se ha utilizado el programa FACTOR (Lorenzo-Seva y Ferrando, 2013).

Se han realizado un análisis descriptivo con el objetivo de conocer las medias y desviación estándar de los ítems correspondientes a las percepciones de los residentes sobre los impactos asociados al turismo activo. La normalidad de los resultados fue examinada a través de los valores de asimetría y curtosis, todos ellos inferiores al criterio recomendando por Chou y Bentler (1995) de 3.0. Para evaluar la fiabilidad de la escala, se han tenido en cuenta medidas como el Alfa de Cronbach, la Fiabilidad Compuesta (FC) y la Varianza Media Extraída (AVE) de cada uno de los factores (Hair, Black, Babin, Anderson y Tatham, 2006).

\section{Resultados}

Tal y como se puede apreciar en la Tabla 1, las diferentes escalas de impactos asociados a la actividad turística físicodeportiva, estas tienen unos índices de validez y fiabilidad aceptable. Asimismo, destaca la escala de impactos políticos administrativos, cuyas valoraciones medias de los ítems se sitúan entre 4,19 y 4,35 puntos de valoración media. Seguidamente, la escala de impactos económicos obtiene una valoración media general de 4,03 $(\mathrm{DT}=, 93)$ sobre 5 puntos.

Además, cabe destacar que los impactos sociales y medioambientales, asociados a los costes del desarrollo del turismo activo en la comunidad, obtienen una valoración general de $2,04(\mathrm{DT}=.99)$ y $2,37(\mathrm{DT}=1,07)$ puntos respectivamente.

Tabla 1. Análisis de las propiedades de los ítems de la escala de percepciones de los residentes sobre los impactos asociados turismo activodeportivo.

\begin{tabular}{|c|c|c|c|}
\hline Cuestionario completo $(\alpha=.79)$ & Media (DT) & A & $\mathrm{C}$ \\
\hline \multicolumn{4}{|l|}{ Impactos Económicos: $\alpha=.79 ; \mathrm{FC}=.79 ; \mathrm{AVE}=.56$} \\
\hline El turismo deportivo aporta una mayor inversión económica a la comunidad & $4.12(.96)$ & -.94 & .40 \\
\hline El turismo deportivo ayuda a mejorar la situación económica para muchos residentes en esta comunidad & $3.89(1.04)$ & -.66 & -.28 \\
\hline El turismo deportivo crea una oportunidad de mercado y atrae inversión extranjera en Gran Canaria & $4.09(.96)$ & -.91 & .28 \\
\hline Total & $4.03(.93)$ & -.84 & .57 \\
\hline \multicolumn{4}{|l|}{ Impactos Culturales: $\alpha=.74 ; \mathrm{FC}=.76 ; \mathrm{AVE}=.52$} \\
\hline El turismo deportivo fomenta una variedad de actividades culturales y eventos para los residentes locales. & $3.65(1.07)$ & -.45 & -.49 \\
\hline $\begin{array}{l}\text { El turismo deportivo ayuda a mantener viva la cultura y ayuda a mantener la identidad étnica de los resi- } \\
\text { dentes locales. }\end{array}$ & $3.33(1.18)$ & -.20 & -.80 \\
\hline $\begin{array}{l}\text { El turismo deportivo ha dado lugar a un mayor intercambio cultural entre turistas y residentes siendo una } \\
\text { experiencia enriquecedora }\end{array}$ & $3.84(1.02)$ & -.62 & -.21 \\
\hline Total & $3.60(.89)$ & -.39 & -.20 \\
\hline \multicolumn{4}{|l|}{ Impactos Politico-administrativos $\alpha=.81 ; \mathrm{FC}=.81 ; \mathrm{AVE}=.58$} \\
\hline La industria del turismo deportivo debe planificarse para el futuro & $4.19(.90)$ & -.96 & .61 \\
\hline Los planes de desarrollo turístico-deportivo deben mejorarse continuamente & $4.35(.86)$ & -1.40 & 1.84 \\
\hline Creo que la isla debe hacer esfuerzo para atraer a más turistas deportivos & $4.23(.99)$ & -.86 & 1.61 \\
\hline Total & $4.26(.78)$ & -1.19 & 2.02 \\
\hline \multicolumn{4}{|l|}{ Impactos Sociales $\alpha=.78 ; \mathrm{FC}=.80 ; \mathrm{AVE}=.58$} \\
\hline El turismo deportivo genera problemas sociales como la delincuencia, consumo de drogas.... & $1.79(1.15)$ & 1.40 & .87 \\
\hline El turismo deportivo crea conflictos entre los residentes y visitantes & $1.97(1.16)$ & 1.03 & .07 \\
\hline Los residentes sufren las consecuencias del turismo deportivo por vivir en una zona de destino turístico & $2.36(1.23)$ & .54 & -.67 \\
\hline Total & $2.04(.99)$ & .97 & .30 \\
\hline
\end{tabular}




\begin{tabular}{|c|c|c|c|}
\hline Cuestionario completo $(\alpha=.79)$ & Media (DT) & A & $\mathrm{C}$ \\
\hline \multicolumn{4}{|l|}{ Impactos Medioambientales $\alpha=.85 ; \mathrm{FC}=.86 ; \mathrm{AVE}=.67$} \\
\hline El turismo deportivo provoca contaminación en el medio ambiente y acelera su deterioro. & $2.21(1.23)$ & .71 & -.54 \\
\hline El turismo deportivo genera contaminación acústica (ruidos), del aire y del agua. & $2.25(1.20)$ & .62 & -.61 \\
\hline El turismo deportivo consume una gran cantidad de recursos naturales (agua, energía...) & $2.65(1.24)$ & .24 & -.91 \\
\hline Total & $2.37(1.07)$ & .49 & -.58 \\
\hline
\end{tabular}

La fiabilidad del modelo se contrastó mediante el alfa de Cronbach, fiabilidad compuesta (FC) y AVE. Como se observa en la tabla, los coeficientes de Alfa de Cronbach oscilan entre .74 y .85, mientras que los de FC para cada factor oscilaron entre .76 y .86 , superiores al criterio recomendado (>.70; Hair et al., 2006). Por otra parte, los valores de AVE oscilaron entre .52 y .67 , también superiores al punto de corte recomendado (>.50; Fornell y Larcker, 1981).

Para valorar la validez discriminante se ha comprobado que todas las correlaciones entre los diversos factores fueran inferiores a .85 (Kline, 2005). Además, se ha comprobado como la raíz cuadrada del AVE es superior a la correlación entre pares de factores (Fornell y Larcker, 1981) criterio que asumían todas las dimensiones.
Tabla 2. Correlaciones en la entre escalas de Impactos

\begin{tabular}{|c|c|c|c|c|c|}
\hline & I.EC. & I.CU. & I.PO & I.SO & I.ME \\
\hline I.EC & 0.75 & & & & \\
\hline I.CU & $.38^{*}$ & 0.72 & & & \\
\hline I.PO & $.44^{*}$ & $.23^{*}$ & 0.76 & & \\
\hline I.SO & $-.20^{*}$ & -.08 & $-.35^{*}$ & 0.76 & \\
\hline I.ME & -.05 & -.10 & $-.17^{*}$ & $.53^{*}$ & 0.82 \\
\hline
\end{tabular}

Por su parte, en la Tabla 3, se muestra la fiabilidad de la escala que mide el apoyo de los residentes hacia el turismo deportivo en Gran Canaria, que a diferencia de las de impactos positivos y negativos, es unidimensional, y está formada por seis ítems. Esta escala será utilizada como variable dependiente en el modelo conceptual. El Alfa de Cronbach general de esta escala es de .81, lo que supone un buen dato de fiabilidad.

Tabla 3. Análisis de las propiedades de los ítems de la escala de percepciones de los residentes sobre apoyo al desarrollo del turismo deportivo.

\begin{tabular}{|c|c|c|c|}
\hline & $\mathrm{M}(\mathrm{DT})$ & A & $\mathrm{C}$ \\
\hline Me gustaría ver a más turistas realizando actividades deportivas en Gran Canaria & $3.98(1.01)$ & -.851 & .246 \\
\hline Apoyo a que el turismo deportivo tenga un papel económico fundamental en Gran Canaria & $4.09(.99)$ & -.995 & .551 \\
\hline $\begin{array}{l}\text { Apoyo al desarrollo de eventos/programas/ servicios turísticos-deportivos (por ejemplo: instalaciones } \\
\text { de recreo, exposiciones, acontecimientos deportivos, eventos, etc.) }\end{array}$ & $4.28(.93)$ & -1.32 & 1.345 \\
\hline En general, yo apoyo el desarrollo del turismo a través del turismo de actividades acuáticas & $4.00(.98)$ & -.852 & .273 \\
\hline En general, yo apoyo el desarrollo del turismo de golf en la isla & $3.21(1.43)$ & -.231 & -1.22 \\
\hline En general, yo apoyo el desarrollo del turismo de naturaleza en la isla (senderismo, cicloturismo...) & $4.35(.92)$ & -1.68 & 2.93 \\
\hline Total & $3.97(.79)$ & -.77 & .392 \\
\hline Alfa de Cronbach & .81 & & \\
\hline
\end{tabular}

Nota: Media (M), desviación típica (DT), asimetría (A) y curtosis (C).

Asimismo, se comprobó la unidimensionalidad de la escala por el cual permite confirmar que los ítems sólo tienen un constructo en común. Este análisis se llevó a cabo con el programa FACTOR, mediante la aplicación del método de
Máxima Verosimilitud, con el método del Análisis Paralelo y la rotación Quartimin directa normalizada, que es la recomendada cuando se espera un solo factor en rotación oblicua. 
Tabla 4. Unidimensionalidad y varianza explicada para la escala de apoyo hacia el turismo deportivo por parte de los residentes de Gran Canaria.

\begin{tabular}{ccccccc}
\hline Dimensión & GFI & RMSR & KMO & Test de esfericidad de Bartlett $(\mathrm{gl})$ & Autovalor & Varianza explicada \% \\
\hline Apoyo & .994 & .043 & 0.85 & $563.1(15)$ & 3.26 & 54.27 \\
\hline
\end{tabular}

Nota. GFI=Índice de Bondad de Ajuste; RMSR=Raíz Media Cuadrática Residual; KMO=Medida de adecuación maestral.

\section{Regresión lineal múltiple}

Se ha realizado un análisis de regresión lineal múltiple con 15 ítems que miden la percepción media de los impactos, tanto positivos como negativos, asociados al turismo deportivos (variables independientes), con la finalidad de conocer cuáles de ellos son los mejores predictores del apoyo a este tipo de actividad económica, como medio de fomento del turismo (variable dependiente). De esta manera, se ha obtenido un modelo predictivo que explica un $47,5 \%$ de la varianza.

Tabla 5. Coeficientes del modelo.

\begin{tabular}{ccccc}
\hline Modelo & $\mathrm{R}$ & $\mathrm{R}$ cuadrado & $\begin{array}{c}\text { R cuadrado } \\
\text { corregida }\end{array}$ & $\begin{array}{c}\text { Error típico de la } \\
\text { estimación }\end{array}$ \\
\hline 1 & .692 & .479 & .475 & .577 \\
\hline
\end{tabular}

Los resultados obtenidos muestran que cuatro de las cinco dimensiones predicen la variable dependiente, siendo la dimensión impactos políticos-administrativos $(\beta=, 365)$, impactos culturales $(\beta=, 246)$ e impactos económicos $(\beta=, 191)$, todas ellas en sentido positivos, las que mayor información aportan a este modelo predictivo.

Tabla 6. Coeficientes de regresión.

\begin{tabular}{llllll}
\hline Escala & Beta & $\mathrm{t}$ & Sig. & Tol & $\mathrm{FIV}$ \\
\hline Impacto Económico & .191 & 5.753 & $.000^{* * *}$ & .733 & 1.365 \\
Impacto Cultural & .246 & 8.641 & $.000^{* * *}$ & .841 & 1.190 \\
Impacto Político-Adminis- & .365 & 10.311 & $.000^{* * *}$ & .714 & 1.400 \\
trativo & -.050 & -1.657 & .098 & .616 & 1.624 \\
Impacto Social & -.114 & -4.381 & $.000^{* * *}$ & .683 & 1.464 \\
\hline Impacto Medioambiental & Nota. "'=p<0.001.; FIV=Factor inflación de la varianza.; Tol=Tolerancia. &
\end{tabular}

\section{Discusion}

Los residentes deben participar en todos los niveles de la planificación, el desarrollo y la ejecución de las actividades turísticas de manera que ayuden y extiendan su hospitalidad a los turistas. Considerando los impactos del turismo activo en el municipio, Medina y Sánchez (2005) señalan que la presencia de actividades físicas y deportivas mejora y diferencia la oferta turística de una zona. Estas actividades pueden llevarse a cabo tanto en el interior como en el litoral, contribuyendo así al desarrollo de este tipo de turismo alternativo.

La literatura sugiere que existe una relación positiva entre los factores asociados con los impactos positivos percibidos y el apoyo de los residentes al desarrollo turístico. De acuerdo con los hallazgos de Stylidis Biran, Sit y Szivas (2014), cuando los residentes adquieren una mejor percepción de los impactos sociales, culturales, ambientales y económicos positivos, apoyan el desarrollo del turismo.

De forma similar a nuestro estudio, Masa'deh, Nasseef, Alshayeb, Ojilat y Alshafiee (2017) descubrieron que existe significación en los impactos medioambientales y económicos con respecto al apoyo al desarrollo turístico, en tanto que la variable impactos sociales no tiene un impacto significativo en el mismo. Además, Dyer et al., (2007) no encontraron ninguna relación entre los impactos sociales negativos y el apoyo de los residentes al desarrollo turístico. Una posible explicación para este hecho sería que algunos residentes excluyen los costos sociales si esperan beneficios económicos (Garau-Vadell, Gutiérrez-Taño y Díaz-Armas, 2018). Debido a que los residentes resaltan los beneficios del sector, estos pueden sobrestimar los impactos positivos y subestimar los impactos negativos asociados a la actividad.

La interrelación entre las variables asociadas a las percepciones de los residentes de las comunidades donde se realizan actividades turísticas y su relación directa con el apoyo al desarrollo de estas actividades en la comunidad ha sido ampliamente estudiada, afirmando la existencia de impactos tanto negativos como positivos (González-García et al., 2016; Parra, Añó, Calabuig y Ayora, 2016).

En este trabajo se destacan los impactos político-administrativos como el factor que tiene mayor impacto en el apoyo de los residentes al desarrollo del turismo deportivo activo. Nunkoo y Gursoy (2016), seńalan que, dado que los trámites de licitación y planificación tienen un componente mínimo de participación ciudadana, el nivel de confianza y percepción que los residentes pueden tener hacia los responsables de organizar y gestionar el sector desempeña un papel significativo, siendo este un factor determinante.

Tras los impactos político-administrativos, los habitantes de Gran Canaria perciben los impactos culturales y económicos como influyentes en la decisión de apoyar el desarrollo del turismo deportivo activo en la comunidad. Algunos estudios han demostrado que las percepciones de los residentes tienden a ser positivas con respecto a los impactos económi- 
cos asociados al sector (Andereck, Valantine, Knopf y Vogt, 2005), mientras que los aspectos socioculturales y ambientales tienen percepciones negativas (Diedrich y García-Buades, 2009).

Para Gran Canaria, los residentes diferencian los impactos sociales de los impactos culturales de manera similar a otras investigaciones (Garau-Vadell et al., 2018). Puede observarse con certeza que los impactos culturales tienen una tendencia positiva, mientras que los impactos sociales tienen una tendencia negativa. En este sentido, cuando la comunidad tiene una actividad turística plenamente integrada, puede obviar algunos de los impactos negativos asociados a ella (Andriotis, 2005), que en el caso concreto de Gran Canaria, los impactos sociales no son significativos con el apoyo del desarrollo del turismo activo en la comunidad, por lo que podría ser una posible explicación de su disociación con respecto a los impactos culturales, así como la indiferencia que los residentes tienen ante los impactos sociales asociados a la actividad turística activa.

En cuanto a los impactos medioambientales asociados a la actividad turístico-deportiva, los grancanarios perciben como significativa su relación con el apoyo al desarrollo de la actividad en la isla. Según un estudio de Bujosa-Bestard y Roselló-Nadal (2007), los residentes de las Islas Baleares destacan los impactos medioambientales como denominador común entre la comunidad.

En términos generales, la mayoría de las investigaciones no han llegado a un consenso sobre qué factores afectan las percepciones de los residentes y qué variables determinan estas percepciones en la comunidad (Almeida-García et al., 2015). Este hecho puede atribuirse a las características específicas de cada población, cada una de las cuales tiene sus propias particularidades, por lo que las percepciones de los residentes variarían (Fredline, 2005). Aunque los resultados pueden tener características en común con otros lugares, si- guen siendo únicos (Ryan, Chaozhi y Zeng, 2011), y por tanto aún se necesita más investigación en esta temática.

\section{Conclusiones}

Como conclusión, se destaca que las dimensiones asociadas al impacto político-administrativo, impacto económico e impacto cultural, son las que mejor predicen el apoyo por parte de los residentes, al desarrollo del turismo activo deportivo. Por otro lado, los impactos medioambientales asociados, disminuyen la predisposición de los residentes a apoyar el desarrollo del turismo activo-deportivo.

Estos datos son de especial relevancia para aquellos gestores y planificadores del sector turístico activo en la comunidad. Con ello, se adquiere información relevante para la mejora de la oferta turística, así como la diversificación del sector en la localidad. Además, se deberían adaptar sus decisiones a las necesidades de la comunidad, lo que aumentará el apoyo hacia la acogida de este tipo de actividades y consecuentemente el éxito turístico deportivo en la localidad.

\section{Limitaciones}

Dada la particularidad de la población, los resultados no son extrapolables a otras poblaciones. Asimismo, el tipo de muestreo establecido podría no ser representativo de las valoraciones globales del resiente.

\section{Futuras líneas de investigación}

En futuras investigaciones, sería interesante analizar las percepciones sobre actividades concretas, y observar si existe algún tipo de preferencias en cuanto al desarrollo de estas en la comunidad.

\section{Referencias}

1. Alguacil, M., Pérez-Campos, C., Berenguer, S. A., \& Boquera, J. (2016). ¿La celebración de un evento promociona la ciudad como destino turístico entre los deportistas?. SPORT TK-Revista EuroAmericana de Ciencias del Deporte, 5, 123-136.

2. Almeida-García, F., Balbuena-Vázquez, A., \& Cortes-Macías, R. (2015). Resident's attitudes towards the impacts of tourism. Tourism Management Perspectives, 13, 33-40.

3. Andereck, K. L., Valentine, K. M., Knopf, R. C., \& Vogt, C. A. (2005). Residents' perceptions of community tourism impacts. Annals of tourism research, 32(4), 1056-1076.

4. Andriotis, K. (2005). Community groups' perceptions of and preferences for tourism development: Evidence from Crete. Journal of Hospitality \& Tourism Research, 29(1), 67-90.

5. Balduck, A. L., Maes, M., \& Buelens, M. (2011). The social impact of the Tour de France: Comparisons of residents' pre-and post-event perceptions. European Sport Management Quarterly, 11(2), 91-113.

6. Bartolomé, A., Ramos, V., \& Rey-Maquieira, J. (2009). Residents' Atti- tudes Towards Diversification Sports Tourism in the Balearics. Tourism Recreation Research, 34(1), 55-65.

7. Bujosa-Bestard, A \& Roselló-Nadal, J. (2007) Modelling environmental attitudes toward tourism. Tourism Management, 28(3), 688-695.

8. Chandralal, K. (2010). Impacts of tourism and community attitudes towards tourism: A case study in Sri Lanka. South Asian Journal of Tourism and Heritage, 3(2), 41-49.

9. Chou, C. P., \& Bentler, P. M. (1995). Estimates and tests in structural equation modeling. R. H. Hoyle (Ed.), Structural equation modeling: Concepts, issues and applications (pp. 37-55). Thousand Oaks, CA: Sage.

10. Delamere, T. A. (2001). Development of a scale to measure resident attitudes toward the social impacts of community festivals, part II: Verification of the scale. Event Management, 7(1), 25-38.

11. Diedrich, A., \& García-Buades, E. (2009). Local perceptions of tourism as indicators of destination decline. Tourism Management, 30(4), $512-521$. 
12. Dumitru, T. (2012). The impact of tourism development on urban environment. Studies in Business \& Economics, 7(3), 160-164.

13. Dyer, P., Gursoy, D., Sharma, B., \& Carter, J. (2007). Structural modeling of resident perceptions of tourism and associated development on the Sunshine Coast, Australia. Tourism management, 28(2), 409-422.

14. Fornell, C., \& Larcker, D. (1981). Evaluating structural equation models with unobservable variables and measurement error. Journal of Marketing Research, 18(1), 39-50.

15. Fredline, E. (2005). Host and guest relations and sport tourism. Sport in Society, 8(2), 263-279.

16. Garau-Vadell, J. B., Gutierrez-Tańo, D. \& Diaz-Armas, R. (2018). Economic Crisis and Residents' Perception of the Impacts of Tourism in Mass Tourism Destinations. Journal of Destination Marketing and Management, 7, 68-75.

17. Gibson, H. (2017). Sport tourism and theory and other developments: some reflections. Journal of Sport \& Tourism, 21(2), 153-158.

18. González-García, R. J., Añó-Sanz, V., Parra-Camacho, D., \& Calabuig-Moreno, F. (2018). Perception of residents about the impact of sports tourism on the community: Analysis and scale-validation. Journal of Physical Education and Sport ${ }^{\oplus}$ (JPES), 18(1), $149-156$.

19. González-García, R. J., Parra, D., González-Serrano, M. H., \& AñóSanz, V. (2016). Los eventos como potenciadores del turismo en Gran Canaria: un análisis desde la percepción social. Revista Intercontinental de Gestão Desportiva, 6(1), 108-120.

20. Gursoy, D., Chi, C. G., \& Dyer, P. (2010). Locals' attitudes toward mass and alternative tourism: The case of Sunshine Coast, Australia. Journal of Travel Research, 49(3), 381-394.

21. Hair, J. F., Black, W. C., Babin, B. J., Anderson, R. E., \& Tatham, R. L. (2006). Multivariate data analysis (6th ed.). Upper Saddle River, NJ: Prentice Hall.

22. Hall, C. M., \& Page, S. J. (2014). The geography of tourism and recreation: Environment, place and space. (4 ed.). New York: Routledge.

23. Higham, J., \& Hinch, T. (2009). Sport and tourism: Globalization, mobility and identity. Oxford: Elsevier Butterworth-Heineman.

24. Hritz, N., \& Ross, C. (2010). The perceived impacts of sport tourism: An urban host community perspective. Journal of Sport Management, 24(2), 119-138.

25. Jaafar, M., Noor, S. M., \& Rasoolimanesh, S. M. (2015). Perception of young local residents toward sustainable conservation programmes: A case study of the Lenggong World Cultural Heritage Site. Tourism Management, 48, 154-163.

26. Karadakis, K. (2012). The Influence of Small-scale Sport Event Impacts on Personal and Community Quality of Life and Support for Sport Event Tourism (Tesis Doctoral). University of Florida, Florida, EEUU.

27. Kline, R. B. (2005). Principles and practice of structural equation modeling (2nd ed.). New York, NY: Guilford.
28. Lorenzo-Seva, U., \& Ferrando, P. J. (2013). FACTOR 9.2: A Comprehensive Program for Fitting Exploratory and Semiconfirmatory Factor Analysis and IRT Models. Applied Psychological Measurement, 37(6), 497-498.

29. Masa'deh, R. E., Nasseef, M. A., Alshayeb, H., Ojilat, J., \& Alshafiee, M. (2017). The Effect of Sport Tourism Management on Support for Tourism Development. Journal of Management and Strategy, 8(3), 20.

30. McGehee, N. G., \& Andereck, K. L. (2004). Factors predicting rural residents' support of tourism. Journal of travel research, 43(2), 131-140.

31. Medina, F. X., \& Sánchez, R. (2005). Actividad físico-deportiva, turismo y desarrollo local en Espańa. PASOS. Revista de Turismo y Patrimonio cultural, 3(1), 97-107.

32. Nunkoo, R., \& Gursoy, D. (2016). Rethinking the role of power and trust in tourism planning. Journal of Hospitality Marketing \& Management, 25(4), 512-522.

33. Nunkoo, R., \& So, K. K. F. (2016). Residents' support for tourism: Testing alternative structural models. Journal of Travel Research, 55(7), 847-861.

34. Parra, D., Añó, V., Calabuig, F., \& Ayora, D. (2016). Percepción de los residentes sobre el legado de la America's Cup. Cuadernos de Psicología del Deporte, 16(1), 309-322.

35. Rasoolimanesh, S. M., Ringle, C. M., Jaafar, M., \& Ramayah, T. (2017). Urban vs. rural destinations: Residents' perceptions, community participation and support for tourism development. Tourism Management, 60, 147-158.

36. Ryan, C., Chaozhi, Z., \& Zeng, D. (2011). The impacts of tourism at a UNESCO heritage site in China-a need for a meta-narrative? The case of the Kaiping Diaolou. Journal of Sustainable Tourism, 19(6), 747-765.

37. Saha, S., \& Yap, G. (2014). The moderation effects of political instability and terrorism on tourism development: A cross-country panel analysis. Journal of Travel Research, 53(4), 509-521.

38. Sánchez-Fernández, P., Salgado-Barandela, J., Rodriguez-Rodriguez, A., \&. Barajas-Alonso, A. (2016). Impacto económico del XXI Campeonato de Espańa “Open” de Invierno Master de Natación de Pontevedra. SPORT TK-Revista EuroAmericana de Ciencias del Deporte, 5, 169-180.

39. Stylidis, D., Biran, A., Sit, J., \& Szivas, E. M. (2014). Residents' support for tourism development: The role of residents' place image and perceived tourism impacts. Tourism Management, 45, 260-274.

40. Ward, C., \& Berno, T. (2011). Beyond social exchange theory: Attitudes toward tourists. Annals of Tourism Research, 38(4), 1556-1569.

41. Weed, M., \& Bull, C. (2012). Sports tourism: Participants, policy and providers. London: Routledge.

42. Woo, E., Uysal, M., \& Sirgy, M. J. (2018). Tourism impact and stakeholders' quality of life. Journal of Hospitality \& Tourism Research, 42(2), 260-286. 